\title{
Art et littérature: le voyage entre texte et image, sous la direction de Jean-Loup Korzilius
}

\section{Luigi Luison}

\section{(2) OpenEdition}

1 Journals

\section{Edizione digitale}

URL: https://journals.openedition.org/studifrancesi/46469

DOI: 10.4000/studifrancesi.46469

ISSN: 2421-5856

\section{Editore}

Rosenberg \& Sellier

\section{Edizione cartacea}

Data di pubblicazione: 1 octobre 2007

Paginazione: 493-494

ISSN: 0039-2944

\section{Notizia bibliografica digitale}

Luigi Luison, «Art et littérature: le voyage entre texte et image, sous la direction de Jean-Loup Korzilius», Studi Francesi [Online], 152 (LI | II) | 2007, online dal 30 novembre 2015, consultato il 24 novembre 2021. URL: http://journals.openedition.org/studifrancesi/46469 ; DOI: https://doi.org/10.4000/ studifrancesi.46469

Questo documento è stato generato automaticamente il 24 novembre 2021.

\section{(c) (1)}

Studi Francesi è distribuita con Licenza Creative Commons Attribuzione - Non commerciale - Non opere derivate 4.0 Internazionale. 


\title{
Art et littérature: le voyage entre texte et image, sous la direction de Jean- Loup Korzilius
}

\author{
Luigi Luison
}

\section{NOTIZIA}

AA. VV., Art et littérature: le voyage entre texte et image, sous la direction de Jean-Loup KoRZILIUS, Amsterdam, New York, Éditions Rodopi, 2006, pp. 314.

1 I saggi raccolti in questo volume affrontano il rapporto tra la parola e l'immagine sotto un duplice aspetto: da una parte, la scrittura e l'arte vengono affrontati parallelamente, dall'altra, la riflessione sul rapporto tra testo e immagine si nutre dello scambio che si produce tra loro. Attraverso l'analisi di alcuni testi, parola e immagine vengono esaminati dagli Autori in maniera congiunta per meglio evidenziare le modalità con cui si rapportano. Tale processo è reso possibile attraverso l'individuazione di un percorso comune di indagine, in grado sia di unire gli esempi presentati dagli Autori, sia di assicurare un prolungamento alla specificità del rapporto testo-immagine, in modo da rispettarne anche il carattere interattivo. Questo percorso comune o tema conduttore, che doveva essenzialmente riflettere il principio della reciprocità e della comunicazione, si concretizza perfettamente nel tema del viaggio e conduce gli Autori in quattro diverse direzioni di indagine: la prima si riferisce allo spostamento fisico, come mezzo di incontro e di evoluzione scaturita dal contatto tra le comunità; la seconda riguarda l'uomo in quanto viaggiatore, che entra in contatto e si integra in nuove realtà; la terza direzione di analisi è quella che evidenzia più chiaramente il rapporto testo-immagine sotto forma di relazione tra il viaggio stesso e la coppia arte/ letteratura (ne consegue che $\mathrm{i}$ viaggi esposti in questo volume sono fortemente associati alle dimensioni visuali e scritturali, passando sia dalla scrittura che dall'arte figurativa); infine, la quarta e ultima direzione si focalizza sull'esame dell'interazione tra i testi scritti presi in esame e le forme scrittorie, al fine di comprendere le diverse 
articolazioni fra immagini e parole, ma soprattutto capire come sono organizzate le reciproche interferenze.

Da un punto di vista testuale, gli articoli raccolti nel volume comprendono giornali di viaggio manoscritti e numerose opere a stampa, mentre da un punto di vista dell'immagine, essi presentano disegni, stampe, pitture e fotografie. L'excursus che si profila fra le mani del lettore comincia dal periodo rinascimentale (epoca in cui in Europa si afferma l'idea dell'uomo come fautore del proprio destino) e arriva sino all'epoca novecentesca; ed è proprio al lettore, il più delle volte, che viene affidato il compito di tirare le fila dei risultati emersi dalle analisi degli Autori.

3 Lungi dall'avere uno statuto conflittuale, la relazione fra testo e immagine è utile, secondo gli Autori, a supportare ideologie e sentimenti suscitati dall'esperienza del viaggio, il cui leitmotiv ricorrente è quello dello spazio, sia fisico che irreale, che si apre davanti agli occhi del viaggiatore. In questa prospettiva, il rapporto tra testo e immagine appare come la metafora dell'esperienza stessa del viaggio, nel senso profondo del termine.

Jean-François KOSTA-THÉfAINE ( $D u$ récit de voyage et de sa mise en image: l'exemple du manuscrit de New York du "Devisement du Monde" de Marco Polo, pp. 31-59) fornisce uno studio sulla relazione tra il testo e l'immagine in un manoscritto in lingua francese del XIV secolo, conservato alla Pierpont Morgan Library di New York. Il documento contiene il racconto dei viaggi di Marco Polo e 41 raffigurazioni. In questo viaggio attraverso il testo e le immagini, l'A. mette in evidenza come le illustrazioni non sempre sono fedeli al resoconto testuale. K. Michelle HEARNE (Le voyage de Gênes: The Queen's Perspective, pp. 61-85) incentra il suo studio su Le voyage de Gênes, testo redatto nel 1508 per la regina Anna di Bretagna. Il documento racconta le imprese militari di Luigi XII, suo sposo, rivolte a reprimere le insurrezioni genovesi del 1506. L'A. descrive le miniature presenti nel manoscritto, che mostrano la regina simbolicamente presente ed esercitante la propria influenza durante la campagna italiana del marito, sebbene la regina non abbia mai lasciato il territorio francese. Secondo l'A., questo espediente è stato utilizzato per rafforzare la posizione della sovrana alla corte e nel gioco politico internazionale. Véronique BOUCHERAT ("Le Journal de voyage" et les dessins de Dürer aux Pays-Bas (1520-1521): des lectures complémentaires, pp. 87-114) descrive il diario tenuto da Albrecht Dürer, il più famoso pittore del rinascimento nordico, durante il soggiorno olandese nel 1520-1521. Analizzando il contenuto testuale e i disegni realizzati dall'artista durante il suo viaggio, l'A. mette in evidenza l'impatto emotivo che questa esperienza olandese ha esercitato sulla personalità del pittore, giungendo alla conclusione che testo e immagine, in questo diario, si completano a vicenda. Frank MULLER (Vers «la belle et féconde Italie, riche en nobles constructions...»: un prince et un architecte allemand en pélerinage artistique à la fin du XVİ̀me siècle, pp. 115-136) racconta il viaggio che il duca Federico di Wurtemberg ha compiuto in Italia tra il 1599 ed il 1600 insieme al suo architetto Heinrich Schickhardt. Quest'ultimo ha raccolto in un diario una serie di disegni dei principali monumenti visitati durante il viaggio. L'interesse di Schickhardt per le decorazioni dei palazzi, delle ville e dei giardini, lo ha portato ad adottare per le sue realizzazioni future a Wurtemberg e Montbéliard i particolari visti durante il soggiorno italiano. Léna WIDERKEHR ( $A u$ fil du Rhin: invitation européenne au voyage entre texte et image de 1570 à 1660 , pp. 137-168) presenta la prima evocazione letteraria del fiume Reno da parte dell'umanista post-luterano Johann Fischart, nello stile del viaggio poetico. L'A. sottolinea la dimensione politica e identitaria del Reno 
come luogo di passaggio e di condivisione culturale. Alla descrizione del viaggio, Fischart associa una ventina di illustrazioni. Contrariamente all'epoca romantica e moderna, l'Umanesimo confina l'interesse nei confronti del Reno quasi esclusivamente al genere poetico. Sophie LINON-CHIPON (Itinéraire d'un «viator pictor»: le regard de Corneille Le Brun dans son "Voyage du Levant" (1674-1693) et la mise en image de Smyrne/Izmir, pp. 169-194) dimostra come, a ogni tappa del suo viaggio in oriente, Corneille Le Brun collezioni e organizzi in un catalogo le immagini di ciò che ha visto e che, secondo lui, bisognerebbe vedere. L'edizione olandese del 1698 e quella francese del 1700 del Voyage $d u$ Levant si potrebbe comparare a un album, in cui a ogni tappa del viaggio corrisponde un'illustrazione. Il gioco che si instaura tra i testi e le immagini che raffigurano l'Altrove, alimenta una sorta di riconquista dell'io. Richard PARISOT (L'œuvre d'art dans les récits de voyage de pasteurs germanophones à la fin du xvIII siècle: jeux d'images et d'influences, pp. 195-215) ricorda che tra il 1770 e il 1800 numerosi pastori germanofoni intrapresero un viaggio di formazione, spesso in qualità di accompagnatori di giovani nobili, e che gran parte di questi pastori redassero racconti in cui è descritto dettagliatamente tutto ciò che videro e rappresero durante il viaggio. Dalla lettura di questi resoconti l'A. evidenzia la questione dell'imitazione dell'opera d'arte all'interno del testo scritto e la sua influenza nella percezione degli stessi autori. Jean-Loup KORSILIUs (Entre imaginaire et réalité: l'italie décrite et peinte chez Arnold Bocklin et Hans von Marées, pp. 217-251) spiega perché nell'arte e nella vita degli artisti Arnold Bocklin e Hans von Marées, la pittura e la scrittura intrattengono relazioni complesse. Ideatori di un alto linguaggio pittorico, questi artisti ne definiscono il rapporto con la scrittura, a partire da un loro interesse spiccatissimo per l'arte italiana. L'A. prende in esame i due pittori come iniziatori del rapporto testo-immagine. Gabriele PADBERG (Heinrich Vogeler: À la recherche du «paradis sur terre», pp. 253-274) presenta il viaggio in Russia del 1923 dell'artista tedesco Heinrich Vogeler. La produzione artistica che scaturisce grazie a questo viaggio è il mezzo attraverso cui l'artista materializza il suo ideale politico. Cercando mezzi di espressione artistica innovatori, Vogeler sviluppa il concetto del tableau complexe, pittura a olio di grandi dimensioni e destinata a servire da modello per manifesti e quadri monumentali. Cosciente, tuttavia, dei limiti di questa tecnica, Vogeler si serve della scrittura come mezzo di espressione complementare, redigendo veri e propri reportages che trattano in maniera analitica i diversi aspetti dell'organizzazione sociale sovietica. Nel caso di Vogeler, testo e immagini non si limitano solo a descrivere la realtà, ma soprattutto la interpretano. Chloé conANT (Les voyages photolittéraires de la plasticienne Sophie Calle: une cartographie plurielle, pp. 275-294) presenta l'arte di Sophie Calle, artista contemporanea che utilizza l'unione di materiali come la fotografia e il testo, per produrre racconti in cui lei è uno dei personaggi. Tutte le opere dell'artista, che si sviluppano da scenari ludici, attribuiscono grande importanza ai luoghi e agli spazi, che diventano, così, palinsesti intimi. Come in un gioco, Calle dimostra che viaggiare nei vari luoghi mitici non è altro che viaggiare all'interno di sé stessi. Annick LANTENOIS et Lue DALL'ARMEllina (Texte et hypertexte. Du voyage à l'errance, pp. 295-314) affrontano il viaggio tra testo e immagine nella sua accezione metaforica, focalizzando la loro attenzione sulla struttura ipertestuale quale frammentazione del racconto in micro-linearità. Secondo gli Autori, oggi la scrittura ipertestuale si sperimenterebbe attraverso tre modalità di esistenza: 1) la scrittura del web; 2) la scrittura che nasce da testi, immagini e suoni, organizzata secondo quello che viene definito tecnicamente il "dispositivo multimediale"; 3) la scrittura che nasce da parole, frasi, testi, immagini e suoni, organizzati e combinati secondo regole riconducibili all'algoritmica. 